\title{
Nocardia acidivorans sp. nov., isolated from soil of the island of Stromboli
}

Correspondence

Peter Kämpfer

peter.kaempfer@agrar.

uni-giessen.de

\author{
Peter Kämpfer, ${ }^{1}$ Birgit Huber, ${ }^{2}$ Sandra Buczolits, ${ }^{2}$ Kathrin Thummes, ${ }^{1}$ \\ Iris Grün-Wollny ${ }^{3}$ and Hans-Jürgen Busse ${ }^{2}$ \\ ${ }^{1}$ Institut für Angewandte Mikrobiologie, Justus-Liebig-Universität Giessen, D-35392 Giessen, \\ Germany \\ ${ }^{2}$ Institut für Bakteriologie, Mykologie und Hygiene, Veterinärmedizinische Universität, A-1210 \\ Wien, Austria \\ ${ }^{3}$ Labor Grün-Wollny, D-35394 Giessen, Germany
}

The genus Nocardia encompasses more than 40 established species of mycolic acid-containing actinomycetes, including recently described species (Albuquerque de Barros et al., 2003; Cui et al., 2005; Iida et al., 2006; Kageyama et al., 2004a, b, c, 2005; Kämpfer et al., 2004; Lee, 2006; Wang et al., 2004; Xu et al., 2005; Yamamura et al., 2005; Yassin \& Brenner, 2005; Yassin et al., 2003; Zhang et al., 2003, 2004). A comprehensive summary of the taxonomy of the genus has been given by Goodfellow et al. (1999).

During the characterization of organisms isolated from different soils, strain GW4-1778 ${ }^{\mathrm{T}}$ was recovered from $1 \mathrm{~g}$ of a soil sample (heated for $10 \mathrm{~s}$ at $100^{\circ} \mathrm{C}$ ) that originated from

The GenBank/EMBL/DDBJ accession number for the $16 \mathrm{~S}$ rRNA gene sequence of strain GW4-1778 ${ }^{\top}$ is AM402972.

An extended neighbour-joining tree based on 16S rRNA gene sequences showing the positions of strain $\mathrm{GW} 4-1778^{\top}$ and other Nocardia species is available as supplementary material with the online version of this paper. the Italian island of Stromboli, after extraction for $2 \mathrm{~h}$ in $10 \mathrm{ml} 0.1 \%(\mathrm{v} / \mathrm{v})$ Tween 80 solution containing $5 \mathrm{mg}$ ampicillin and dilution on mannitol-rifampicin agar [containing $1^{-1}$ : mannitol, $10 \mathrm{~g}$; yeast extract, $7 \mathrm{~g}$; Casamino acids, $2 \mathrm{~g}$; peptone (Bacto), $1 \mathrm{~g} ; \mathrm{NaCl}, 1 \mathrm{~g} ; \mathrm{CaCO}_{3}, 0.2 \mathrm{~g}$; nystatin, $100 \mathrm{mg}$; and rifampicin, $5 \mathrm{mg}$ ] for 6 weeks at $27^{\circ} \mathrm{C}$. The strain was maintained on Medium 65 (DSMZ, http://www. dsmz.de/media/med065.htm) at $25^{\circ} \mathrm{C}$ and showed on this medium an orange-coloured substrate mycelium. Yellowishwhite aerial hyphae were formed.

Cells of strain GW4-1778 ${ }^{\mathrm{T}}$ were Gram-positive using the staining procedure described by Gerhardt et al. (1994). Cell morphology was observed under a Zeiss light microscope at $\times 1000$, with cells grown for 3 days at $25^{\circ} \mathrm{C}$ on Medium 65 . The 16S rRNA gene was analysed as described by Kämpfer et al. (2003). Phylogenetic analysis was performed using the software package MEGA (Molecular Evolutionary Genetics Analysis) version 2.1 (Kumar et al., 2001) after multiple alignment of data using CLUSTAL_X (Thompson et al., 1997). 
Distances (distance options according to the Kimura two-parameter model) and clustering with the neighbourjoining method and maximum-parsimony (data not shown) were performed by using bootstrap values based on 1000 replications. The sequenced length of the $16 \mathrm{~S}$ rRNA gene of strain GW4-1778 ${ }^{\mathrm{T}}$ was 1493 bp. Sequence similarity calculations (based on $1299 \mathrm{nt}$ ) after a neighbour-joining analysis (Fig. 1 and Supplementary Fig. S1 available in IJSEM Online) indicated that the closest relatives of strain GW4$1778^{\mathrm{T}}$ were Nocardia pseudobrasiliensis (GenBank accession no. DQ659914; 98.6\%), Nocardia nova (Z36930; 98.6\%), Nocardia niigatensis (AB092563; $98.4 \%$ ), Nocardia jiangxiensis (AY639902; 98.0\%), Nocardia uniformis (Z46752; 98.0\%) and Nocardia miyunensis (AY639901; 97.8\%). Lower sequence similarities $(<97.5 \%)$ were found with all other established species of the genus Nocardia.

Analysis of menaquinones was done as described by Tindall (1990) and Altenburger et al. (1997) and that of polar lipids as described by Ventosa et al. (1993). Strain GW4-1778 exhibited a quinone system with the predominant compound MK-8 $\left(\mathrm{H}_{4}, \omega-\mathrm{cycl}\right)(99.5 \%)$ and traces of MK-8 $\left(\mathrm{H}_{4}\right)$. This trait is characteristic of members of the genus Nocardia (Goodfellow et al., 1999). The polar lipid profile of strain GW4-1778 ${ }^{\mathrm{T}}$ consisted of the predominant compound diphosphatidylglycerol, moderate amounts of phosphatidylethanolamine, phosphatidylinositol, two phosphatidylinositol mannosides, an unknown polar lipid and trace amounts of two unknown lipids. Traces of phosphatidylglycerol may be present, as assumed by the presence of a weak spot showing the chromatographic behaviour for this lipid, but could not be stained with molybdenum blue, which is used to indicate the presence of phosphate groups (data not shown). However, the lack or presence only of trace amounts of phosphatidylglycerol has been reported for several nocardiae (Minnikin et al. 1977). The presence of only one aminolipid, phosphatidylethanolamine, demonstrates that strain GW4-1778 ${ }^{\mathrm{T}}$ exhibits phospholipid type II (Lechevalier et al., 1977) and thus this polar lipid profile is in accordance with the characteristics of the genus Nocardia.

Fatty acid analysis was performed according to Kämpfer \& Kroppenstedt (1996). The fatty acid profile of strain GW4$1778^{\mathrm{T}}$ given in Table 1 was similar to those of the other closely related species, but also showed pronounced quantitative differences.

Detailed results of the physiological characterization are given in Table 2 and the species description, using methods as described previously (Kämpfer et al., 1991). DNA-DNA hybridization experiments were performed using labelled DNA from strain GW4-1778 ${ }^{\mathrm{T}}$ and type strains of other Nocardia species using the method described by Ziemke et al. (1998) except that, for nick translation, $2 \mu \mathrm{g}$ of DNA was labelled during a $3 \mathrm{~h}$ incubation at $15^{\circ} \mathrm{C}$. The DNADNA hybridization values obtained were as follows: $N$. pseudobrasiliensis DSM $44290^{\mathrm{T}}(46.1 \%)$, N. nova DSM $44481^{\mathrm{T}}(62.6 \%), N$. jiangxiensis DSM $17684^{\mathrm{T}}(50.9 \%), N$. miyunensis DSM $17685^{\mathrm{T}}(41.7 \%)$, N. niigatensis DSM $44670^{\mathrm{T}}(11.0 \%)$ and N. uniformis DSM $43136^{\mathrm{T}}(21.0 \%)$.

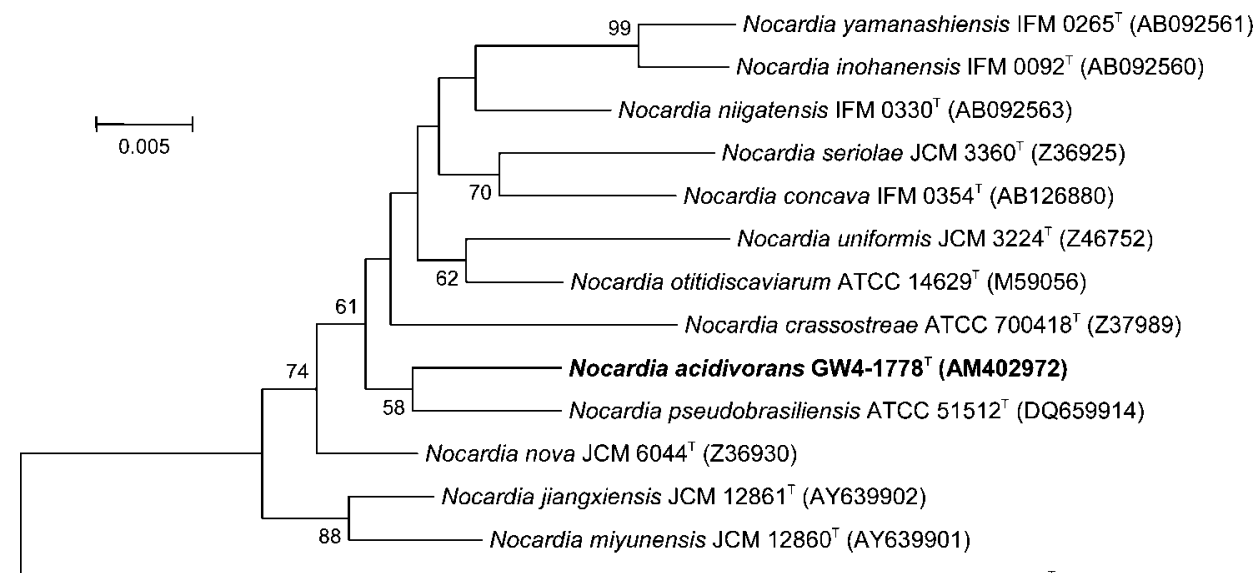

Gordonia terrae ATCC $25594^{\top}$ (X81922)

Fig. 1. Phylogenetic analysis of selected Nocardia species most closely related to strain GW4-1778 ${ }^{\top}$, based on $16 \mathrm{~S}$ rRNA gene sequences available from GenBank/EMBL/DDBJ (accession numbers are given in parentheses), constructed after multiple alignment of data by using CLUSTAL_X (Thompson et al., 1997). Distances (distance options according to the Kimura two-parameter model) and clustering with the neighbour-joining method were performed by using the software package MEGA (Molecular Evolutionary Genetics Analysis) version 2.1 (Kumar et al., 2001). Bootstrap percentages based on 1000 replications are given at branch points. Bar, 0.005 nucleotide substitutions per nucleotide position. An extended neighbourjoining tree is available as Supplementary Fig. S1 in IJSEM Online. 
Table 1. Major fatty acid compositions (\%) of strain GW4-1778 ${ }^{\top}$ and type strains of species of the genus Nocardia grouped in the same cluster on the basis of $16 \mathrm{~S}$ rRNA gene sequence similarity (see Fig. 1)

Strains: 1, GW4-1778 ${ }^{\mathrm{T}}$ (Nocardia acidivorans sp. nov.); 2, N. jiangxiensis DSM $17684^{\mathrm{T}} ; 3$, N. miyunensis DSM $17685^{\mathrm{T}} ; 4$, N. uniformis DSM $43136^{\mathrm{T}}$; 5, N. pseudobrasiliensis DSM $44290^{\mathrm{T}}$; 6, N. nova DSM $44481^{\mathrm{T}} ; 7, \mathrm{~N}$. niigatensis DSM $44670^{\mathrm{T}}$. All strains were grown on trypticase soy broth agar at $28^{\circ} \mathrm{C}$ for 7 days prior to fatty acid analysis. For unsaturated fatty acids, the position of the double bond is located by counting from the methyl $(\omega)$ end of the carbon chain; cis and trans isomers are indicated by the suffixes $c$ and $t$, respectively. -, Not detected.

\begin{tabular}{|c|c|c|c|c|c|c|c|}
\hline Fatty acid & 1 & 2 & 3 & 4 & 5 & 6 & 7 \\
\hline \multicolumn{8}{|l|}{ Saturated fatty acids: } \\
\hline $\mathrm{C}_{13: 0}$ & 0.7 & - & - & - & - & - & - \\
\hline $\mathrm{C}_{14: 0}$ & 1.9 & 0.7 & - & 1.0 & 0.4 & 1.0 & 1.6 \\
\hline $\mathrm{C}_{15: 0}$ & 20.3 & 2.1 & 6.0 & 5.7 & 1.4 & 1.1 & 5.0 \\
\hline $\mathrm{C}_{16: 0}$ & 11.7 & 35.7 & 38.2 & 33.4 & 40.2 & 37.7 & 32.1 \\
\hline $\mathrm{C}_{17: 0}$ & 6.6 & 2.7 & 2.9 & 2.3 & 1.9 & 1.9 & 4.2 \\
\hline $\mathrm{C}_{18: 0}$ & - & 2.1 & 0.8 & 0.7 & 8.6 & 7.2 & 3.0 \\
\hline $\mathrm{C}_{20: 0}$ & - & 0.5 & - & - & - & - & - \\
\hline \multicolumn{8}{|l|}{ Unsaturated fatty acids: } \\
\hline $\mathrm{C}_{15: 1} \omega 5 c$ & 4.4 & - & 1.1 & - & - & - & 1.5 \\
\hline $\mathrm{C}_{16: 1} \omega 9 c$ & - & 0.7 & - & - & - & - & - \\
\hline $\mathrm{C}_{17: 1} \omega 9 c$ & - & - & - & - & - & - & - \\
\hline $\mathrm{C}_{17: 1} \omega 8 c$ & 13.8 & 1.8 & 4.9 & 2.4 & 0.8 & - & 4.0 \\
\hline $\mathrm{C}_{17: 1} \omega 5 c$ & 0.6 & 1.0 & 1.0 & - & - & - & - \\
\hline $\mathrm{C}_{18: 1} \omega 9 c$ & 5.7 & 13.1 & 11.5 & 19.0 & 6.3 & 15.6 & 12.1 \\
\hline $\mathrm{C}_{20: 4} \omega 6,9,12,15 c$ & - & - & - & - & - & - & 0.7 \\
\hline $\mathrm{C}_{20: 2} \omega 6,9 c$ & - & - & - & 1.1 & - & - & - \\
\hline $\mathrm{C}_{20: 1} \omega 9 c$ & - & 2.2 & - & - & - & - & - \\
\hline Summed feature $2^{\star}$ & - & - & - & - & - & - & - \\
\hline Summed feature $3^{\star}$ & 12.1 & 6.7 & 12.3 & 16.4 & 14.4 & 20.0 & 16.1 \\
\hline Summed feature $5^{\star}$ & - & - & - & - & - & - & - \\
\hline Summed feature $6^{*}$ & 2.8 & 1.5 & 0.1 & 2.1 & - & - & 1.7 \\
\hline 10-Methyl $\mathrm{C}_{16: 0}$ & - & - & - & - & - & - & 0.8 \\
\hline 10-Methyl $\mathrm{C}_{17: 0}$ & 11.3 & 1.0 & 3.4 & 1.0 & 0.9 & - & 2.5 \\
\hline 10-Methyl $\mathrm{C}_{18: 0}(\mathrm{TSBA}) \dagger$ & 6.8 & 13.3 & 17.0 & 13.9 & 24.8 & 14.8 & 14.5 \\
\hline 10-Methyl $C_{19: 0}$ & 1.0 & - & - & 1.0 & - & - & - \\
\hline Unknown 14.959 & - & 14.1 & - & - & - & 0.7 & - \\
\hline
\end{tabular}

${ }^{*}$ Summed features are groups of two or three fatty acids that cannot be separated by GLC with the MIDI system. Summed feature 3 contains $\mathrm{C}_{16: 1} \omega 7 c$ and/or $\mathrm{C}_{15: 0}$ iso 2-OH. Summed feature 6 contains $\mathrm{C}_{19: 1} \omega 11 c$ and/or $\mathrm{C}_{19: 1} \omega 9 c$.

$\dagger$ TSBA, Tuberculostearic acid.

$\ddagger$ Unknown fatty acid that has no name listed in the peak library file of the MIDI system and therefore cannot be identified; it is referred to by its equivalent chain length.

\section{Description of Nocardia acidivorans sp. nov.}

Nocardia acidivorans (a.cid.i.vo'rans. N.L. n. acidum an acid; L. v. vorare to devour; N.L. part. adj. acidivorans acid-devouring).

Forms a light orange-coloured vegetative mycelium. Aerial mycelium is yellowish-white. Gram-positive and oxidasepositive, showing an oxidative metabolism. Good growth occurs on nutrient agar and Medium 65 at $25-30^{\circ} \mathrm{C}$. The menaquinone system contains predominantly MK- $8\left(\mathrm{H}_{4}\right.$, $\omega$-cycl) and traces of MK- $8\left(\mathrm{H}_{4}\right)$. The polar lipid profile consists of the predominant compound diphosphatidylglycerol, moderate amounts of phosphatidylethanolamine, phosphatidylinositol, two phosphatidylinositol mannosides, an unknown polar lipid and trace amounts of two unknown lipids. Major fatty acids are $\mathrm{C}_{15: 0}, \mathrm{C}_{16: 0}, \mathrm{C}_{17: 1} \omega 8 \mathrm{c}$ and 10-methyl $\mathrm{C}_{17: 0}$. Carbon source utilization and hydrolysis of chromogenic substrates (including differential characteristics) are given in Table 2. 
Table 2. Physiological properties that distinguish strain GW4-1778 ${ }^{\top}$ from the type strains of the most closely related Nocardia species

Strains: 1, GW4-1778 ${ }^{\mathrm{T}}$ (Nocardia acidivorans sp. nov.); 2, N. jiangxiensis DSM $17684^{\mathrm{T}} ; 3$, N. miyunensis DSM $17685^{\mathrm{T}}$; 4, N. uniformis DSM 43136 ${ }^{\mathrm{T}}$; 5, N. pseudobrasiliensis DSM 44290 ${ }^{\mathrm{T}}$; 6, N. nova DSM $44481^{\mathrm{T}}$; 7, N. niigatensis DSM $44670^{\mathrm{T}}$. +, Positive; -, negative; $(+)$, weakly positive; pNA, p-nitroanilide; pNP, $p$-nitrophenyl. All strains are positive for hydrolysis of aesculin, bis-pNP phosphate, pNP phenylphosphonate and L-alanine pNA and utilization of $\mathrm{N}$-acetyl-D-glucosamine, D-glucose, D-ribose, inositol, glutarate and DL-3-hydroxybutyrate. All strains are negative for hydrolysis of pNP $\beta$-D-glucuronide, pNP phosphorylcholine, L-glutamate- $\gamma$-3-carboxy pNA, L-proline pNA and utilization of gluconate, maltose, D-xylose, adonitol, trans-aconitate, adipate, itaconate, DL-lactate, mesaconate, oxoglutarate, suberate, L-aspartate, L-ornithine, L-phenylalanine, L-tryptophan, 3-hydroxybenzoate, 4-hydroxybenzoate and phenylacetate.

\begin{tabular}{|c|c|c|c|c|c|c|c|}
\hline Characteristic & 1 & 2 & 3 & 4 & 5 & 6 & 7 \\
\hline \multicolumn{8}{|l|}{ Hydrolysis of: } \\
\hline pNP $\beta$-D-galactopyranoside & - & - & - & $(+)$ & - & - & - \\
\hline pNP $\alpha$-D-glucopyranoside & - & + & - & + & + & + & + \\
\hline pNP $\beta$-D-glucopyranoside & + & + & - & + & + & + & + \\
\hline pNP $\beta$-D-xylopyranoside & + & + & - & + & + & + & + \\
\hline 2-Deoxythymidine-5' -pNP phosphate & - & - & - & + & - & - & $(+)$ \\
\hline \multicolumn{8}{|l|}{ Utilization of: } \\
\hline$N$-Acetyl-D-galactosamine & $(+)$ & $(+)$ & - & $(+)$ & $(+)$ & - & - \\
\hline L-Arabinose & $(+)$ & + & + & - & - & - & - \\
\hline$p$-Arbutin & - & + & + & + & - & - & - \\
\hline D-Cellobiose & - & + & + & - & - & - & - \\
\hline D-Fructose & - & + & + & + & $(+)$ & - & - \\
\hline D-Galactose & $(+)$ & $(+)$ & $(+)$ & - & $(+)$ & $(+)$ & $(+)$ \\
\hline D-Mannose & $(+)$ & + & - & - & - & - & - \\
\hline$\alpha$-D-Melibiose & - & + & + & - & - & - & - \\
\hline L-Rhamnose & - & + & + & - & - & - & - \\
\hline Sucrose & - & + & + & - & - & - & - \\
\hline Salicin & - & - & - & + & - & - & - \\
\hline D-Trehalose & - & - & - & - & $(+)$ & - & - \\
\hline Maltitol & - & + & - & - & - & - & - \\
\hline D-Mannitol & + & + & - & $(+)$ & - & - & - \\
\hline D-Sorbitol & - & - & - & - & $(+)$ & - & - \\
\hline Putrescine & + & - & - & - & - & - & - \\
\hline Acetate & + & - & - & - & - & - & - \\
\hline Propionate & + & - & - & - & - & - & - \\
\hline cis-Aconitate & + & - & - & - & - & - & - \\
\hline 4-Aminobutyrate & - & - & + & - & - & - & - \\
\hline Azelate & + & - & - & - & - & $(+)$ & $(+)$ \\
\hline Citrate & + & - & - & - & $(+)$ & - & - \\
\hline Fumarate & + & $(+)$ & + & + & + & + & $(+)$ \\
\hline L-Malate & - & - & + & + & + & $(+)$ & $(+)$ \\
\hline Pyruvate & $(+)$ & - & - & - & - & - & - \\
\hline L-Alanine & $(+)$ & $(+)$ & + & $(+)$ & $(+)$ & - & - \\
\hline$\beta$-Alanine & - & - & + & - & - & - & - \\
\hline L-Histidine & $(+)$ & - & - & - & - & - & - \\
\hline L-Leucine & - & - & $(+)$ & - & $(+)$ & - & - \\
\hline L-Proline & - & - & + & $(+)$ & $(+)$ & - & - \\
\hline L-Serine & $(+)$ & - & - & - & - & - & - \\
\hline
\end{tabular}


The type strain, GW4-1778 ${ }^{\mathrm{T}} \quad\left(=\mathrm{CCUG} 53410^{\mathrm{T}}=\mathrm{CIP}\right.$ $109315^{\mathrm{T}}=$ DSM $45049^{\mathrm{T}}$ ), was isolated from soil of Stromboli, Italy.

\section{References}

Albuquerque de Barros, E. V. S., Manfio, G., Maitan, V. R., Bataus, L. A. M., Kim, S. B., Maldonado, L. A. \& Goodfellow, M. (2003). Nocardia cerradoensis sp. nov., a novel isolate from Cerrado soil in Brazil. Int J Syst Evol Microbiol 53, 29-33.

Altenburger, P., Kämpfer, P., Akimov, V. N., Lubitz, W. \& Busse, H.-J. (1997). Polyamine distribution in actinomycetes with group $B$ peptidoglycan and species of the genera Brevibacterium, Corynebacterium, and Tsukamurella. Int J Syst Bacteriol 47, 270-277.

Cui, Q., Wang, L., Huang, Y., Liu, Z. \& Goodfellow, M. (2005). Nocardia jiangxiensis sp. nov. and Nocardia miyuensis sp. nov., isolated from acidic soils. Int J Syst Evol Microbiol 55, 1921-1925.

Gerhardt, P., Murray, R. G. E., Wood, W. A. \& Krieg, N. R. (editors) (1994). Methods for General and Molecular Bacteriology. Washington, DC: American Society for Microbiology.

Goodfellow, M., Isik, K. \& Yates, E. (1999). Actinomycete systematics: an unfinished synthesis. Nova Acta Leopoldina NF80 (312), 47-82.

lida, S., Kageyama, A., Yazawa, K., Uchiyama, N., Toyohara, T., Chohnabayashi, N., Suzuki, S.-i., Nomura, F., Kroppenstedt, R. M. \& Mikami, Y. (2006). Nocardia exalbida sp. nov., isolated from Japanese patients with nocardiosis. Int J Syst Evol Microbiol 56, 1193-1196.

Kageyama, A., Yazawa, K., Mukai, A., Kinoshita, M., Takata, N., Nishimura, K., Kroppenstedt, R. M. \& Mikami, Y. (2004a). Nocardia shimofusensis sp. nov., isolated from soil, and Nocardia higoensis sp. nov., isolated from a patient with lung nocardiosis in Japan. Int J Syst Evol Microbiol 54, 1927-1931.

Kageyama, A., Yazawa, K., Mukai, A., Kohara, T., Nishimura, K., Kroppenstedt, R. M. \& Mikami, Y. (2004b). Nocardia araoensis sp. nov. and Nocardia pneumoniae sp. nov., isolated from patients in Japan. Int J Syst Evol Microbiol 54, 2025-2029.

Kageyama, A., Yazawa, K., Nishimura, K. \& Mikami, Y. (2004c). Nocardia inohanensis sp. nov., Nocardia yamanashiensis sp. nov. and Nocardia niigatensis sp. nov., isolated from clinical specimens. Int J Syst Evol Microbiol 54, 563-569.

Kageyama, A., Yazawa, K., Taniguchi, H., Chibana, H., Nishimura, K., Kroppenstedt, R. M. \& Mikami, Y. (2005). Nocardia concava sp. nov., isolated from Japanese patients. Int J Syst Evol Microbiol 55, 2081-2083.

Kämpfer, P. \& Kroppenstedt, R. M. (1996). Numerical analysis of fatty acid patterns of coryneform bacteria and related taxa. Can J Microbiol 42, 989-1005.

Kämpfer, P., Steiof, M. \& Dott, W. (1991). Microbiological characterisation of a fuel-oil contaminated site including numerical identification of heterotrophic water and soil bacteria. Microb Ecol 21, 227-251.
Kämpfer, P., Dreyer, U., Neef, A., Dott, W. \& Busse, H.-J. (2003). Chryseobacterium defluvii sp. nov., isolated from wastewater. Int J Syst Evol Microbiol 53, 93-97.

Kämpfer, P., Buczolits, S., Jäckel, U., Grün-Wollny, I. \& Busse, H. J. (2004). Nocardia tenerifensis sp. nov. Int J Syst Evol Microbiol 54, 381-383.

Kumar, S., Tamura, K., Jakobsen, I. B. \& Nei, M. (2001). MEGA2: Molecular Evolutionary Genetics Analysis software. Bioinformatics 17, 1244-1245.

Lechevalier, M. P., De Bièvre, C. \& Lechevalier, H. (1977). Chemotaxonomy of aerobic actinomycetes: phospholipid composition. Biochem Syst Ecol 5, 249-260.

Lee, S. D. (2006). Nocardia jejuensis sp. nov., a novel actinomycete isolated from a natural cave on Jeju Island, Republic of Korea. Int J Syst Evol Microbiol 56, 559-562.

Minnikin, D. E., Patel, P. V., Alshamaony, L. \& Goodfellow, M. (1977). Polar lipid composition in the classification of Nocardia and related bacteria. Int J Syst Bacteriol 27, 104-117.

Thompson, J. D., Gibson, T. J., Plewniak, F., Jeanmougin, F. \& Higgins, D. G. (1997). The CLUSTAL_X windows interface: flexible strategies for multiple sequence alignment aided by quality analysis tools. Nucleic Acids Res 25, 4876-4882.

Tindall, B. J. (1990). Lipid composition of Halobacterium lacusprofundi. FEMS Microbiol Lett 66, 199-202.

Ventosa, A., Marquez, M. C., Kocur, M. \& Tindall, B. J. (1993). Comparative study of "Micrococcus sp." strains CCM 168 and CCM 1405 and members of the genus Salinicoccus. Int J Syst Bacteriol 43, 245-248.

Wang, L., Zhang, Y., Huang, Y., Maldonado, L. A., Liu, Z. \& Goodfellow, M. (2004). Nocardia pigrifrangens sp. nov., a novel actinomycete isolated from a contaminated agar plate. Int J Syst Evol Microbiol 54, 1683-1686.

Xu, P., Li, W.-J., Tang, S.-K., Jiang, Y., Chen, H.-H., Xu, L.-H. \& Jiang, C.-L. (2005). Nocardia polyresistens sp. nov. Int J Syst Evol Microbiol 55, 1465-1470.

Yamamura, H., Hayakawa, M., Nakagawa, Y., Tamura, T., Kohno, T., Komatsu, F. \& limura, Y. (2005). Nocardia takedensis sp. nov., isolated from moat sediment and scumming activated sludge. Int J Syst Evol Microbiol 55, 433-436.

Yassin, A. F. \& Brenner, S. (2005). Nocardia elegans sp. nov., a member of the Nocardia vaccinii clade isolated from sputum. Int J Syst Evol Microbiol 55, 1505-1509.

Yassin, A. F., Sträubler, B., Schumann, P. \& Schaal, K. P. (2003). Nocardia puris sp. nov. Int J Syst Evol Microbiol 53, 1595-1599.

Zhang, J., Liu, Z. \& Goodfellow, M. (2003). Nocardia caishijiensis sp. nov., a novel soil actinomycete. Int J Syst Evol Microbiol 53, 999-1004.

Zhang, Y., Liu, Z. \& Goodfellow, M. (2004). Nocardia xishanensis sp. nov., a novel actinomycete isolated from soil. Int J Syst Evol Microbiol 54, 2301-2305.

Ziemke, F., Höfle, M. G., Lalucat, J. \& Rosselló-Mora, R. (1998). Reclassification of Shewanella putrefaciens Owen's genomic group II as Shewanella baltica sp. nov. Int J Syst Bacteriol 48, 179-186. 